\title{
PERFORMANCE OF A BEAM MONITOR IN THE FERMILAB TEVATRON USING SYNCHROTRON LIGHT ${ }^{*} \dagger$
}

\author{
H. W. K. Cheung, A. Hahn, A. Xiao, FNAL, Batavia, IL 60510, USA
}

\section{Abstract}

Synclite, the beam monitor in the Fermilab Tevatron using synchrotron light is described. The calibration, monitoring and performance of the system is discussed. Observation of some effects of long range beam-beam interactions seen in the beam monitor will be presented as well as a measurement of DC beam in the Tevatron.

\section{INTRODUCTION}

Synchrotron light emitted at the edge of a dipole magnet has a significantly larger intensity in shorter wavelengths than that emitted in an uniform field [1,2]. Blue light emitted from the $\sim 5 \mathrm{~cm}$ turn-on region of a Tevatron dipole can be used to measure the 2-dimensional transverse profile of the beam $[2,3]$. Synchrotron light emitted by protons (antiprotons) from the upstream edge of a 6 (3) meter superconducting magnet is deflected out of the beam pipe by a movable aluminized flat mirror through a quartz window into a telescope assembly. The light is separated from the particle beam by $2.6(1.0) \mathrm{cm}$ at the pick-off point.

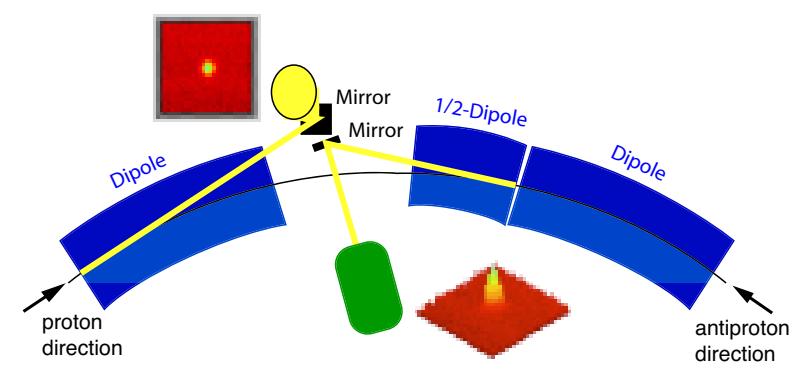

Figure 1: Synclite schematic showing radiation point locations for protons and antiprotons.

The proton and antiproton telescope systems each consist of a single lens and a narrow band blue filter focusing onto the photocathode of a single stage MCP image intensifier. The image on the intensifier phosphor screen is viewed by a CID camera which in turn is captured using a video frame grabber giving an image with $640 \times 480$ (horizontal $\times$ vertical) pixels each with 8 -bits of intensity data. Each pixel in the proton (antiproton) image corresponds to 57 (83) $\mu \mathrm{m}$ at the object plane. The beam transverse profiles are Gaussian with a sigma of about $0.6 \mathrm{~mm}$, and the diffraction resolution is about $0.2 \mathrm{~mm}(0.1 \mathrm{~mm})$ in the horizontal (vertical). More details on the physics of

\footnotetext{
${ }^{*}$ Work supported by the Universities Research Association, Inc., under contract DE-AC02-76CH03000 with the U.S. Dept. of Energy.

$\dagger$ Presented at the 2003 Particle Accelerator Conference, May 12-16 2003, Portland, Oregon, U.S.A.
}

the radiation, the Tevatron dipoles and the telescope optical system can be found in earlier reports[2,3].

\section{Timing and Gain}

Both the proton and antiproton beams in the Tevatron are divided into 36 bunches. Each proton (antiproton) bunch contains an average number of about $210 \times 10^{9}\left(25 \times 10^{9}\right)$ particles at the beginning of the store and have the time structure illustrated in Figure 2(b). The bunches are in 3 trains of 12 bunches each where each bunch is separated by $396 \mathrm{~ns}$. The last bunch of one train is separated from the beginning of the next by $2640 \mathrm{~ns}$, these gaps are called the beam abort gaps. Although the CID camera integrates over about $30 \mathrm{~ms}$, the profile for each bunch can be measured by gating the photocathode so that the image intensifier is only sensitive while a particular bunch is illuminating the photocathode.

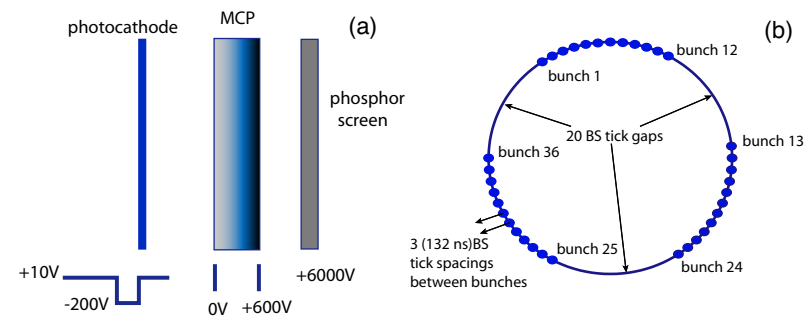

Figure 2: (a) Schematic of image intensifier showing the typical high voltages used; (b) Schematic of beam in the Tevatron illustrating the time structure for the 36 proton bunches. The 36 antiproton bunches have a similar time structure.

The image intensifier consists of a photocathode, microchannel plate and phosphor screen, see Figure 2(a). The gain of the image intensifier varies across its surface. It is calibrated by the use of a UV lamp.

\section{Data Acquisition and Image Analysis}

The Synclite system is controlled by a LabVIEW program running on a Mac, and the analysis of the video image is also done within LabVIEW. Sidebands in the image are used as an estimate of the background to give a background subtracted image. The background subtracted image is either fit with a 2-dimensional Gaussian plus a linear background function, or for a faster fit, the data is projected to give vertical and horizontal profiles and then a 1-dimensional Gaussian plus a linear background function is used in the fits. If the transverse profile is elliptical, 
the full 2-dimensional fit is useful to get the angle of any rotation that might exist.

The measured beam positions, sigmas and the calculated vertical and horizontal emittances are remotely accessible via Acnet and is also logged into a circular buffer and less frequently into a permanent storage for Shot Data Analysis (SDA) [4].

\section{PERFORMANCE}

\section{Calibration and Monitoring}

Calibration of the Synclite system is important in getting accurate measures of the beam parameters. The distance scale, image resolution and any rotation in the system must be known. The scale and image resolution can be calculated and measured on the bench. The distance scale and any rotation of the system has also been measured in situ by producing closed-orbit 3-bumps. The beam is bumped vertically or horizontally by a calculated amount and the motion of the beam measured in Synclite. Figure 3 shows the data from such a beam study and Figure 4 shows for protons the measured versus calculated expected beam displacements.

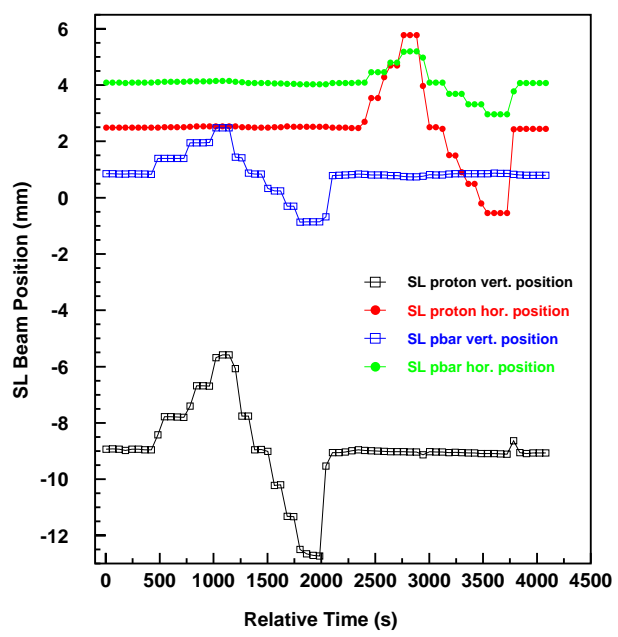

Figure 3: The horizontal and vertical locations of the proton and antiproton beams as measured by Synclite versus time, for the time period when the beams were bumped vertically then horizontally.

The beam bump study results showed that both the proton and antiproton Synclite systems are not rotated with respect to the Tevatron. The proton distance scale obtained by the beam bump study agrees with the expected scale by $\sim 10 \%$, which is the expected uncertainty in the lattice functions. On the other hand the antiproton scale was found to be inconsistent.

The image resolution was checked by scraping the beam with a collimator and studying the beam image size as a function of intensity [5]. It was also studied by comparing
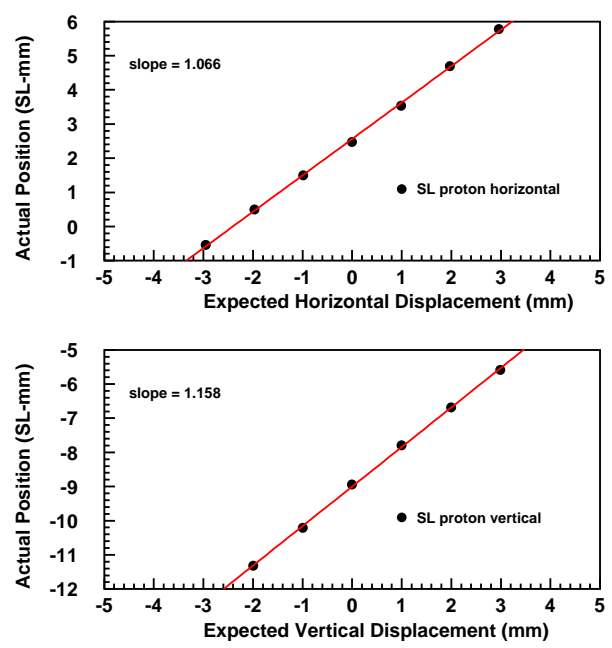

Figure 4: The horizontal and vertical proton beam displacements as measured by Synclite versus the calculated expected displacements.

the beam size as seen by Synclite to that measured in the Flying Wire system [6,7]. The performance of the proton Synclite system is as expected. However, although the antiproton Synclite system is giving useful results, it has an unexpectedly large resolution, this together with the scale is still under study and must be resolved to improve the performance of the antiproton Synclite system.

Monitoring the status and results of the Synclite system is an important aspect of providing a reliable instrument. The status and results from Synclite are obtained via a Java program and displayed on a web page using an automatically run PERL script [8]. If the Synclite system is down or has incorrect settings, notification emails are sent in additional to a message on the status web page.

\section{Long Range Beam-Beam Effects}

The Synclite system can be used to study beam physics. For example one effect of long range beam-beam interactions can be seen in the Synclite measurements. The top plots in Figure 5 show the horizontal positions of all the 36 antiproton bunches averaged over various time periods after the start of a collider store. It can be seen that a 3-fold symmetry is seen where each train of 12 bunches is spread over about $40 \mu \mathrm{m}$. This structure exists because the start of each train interacts with more protons than the end of the train. A similar 3-fold structure is seen in the proton horizontal positions but the 12 bunches of each train are spread over a much smaller range of about $5 \mu \mathrm{m}$. This smaller effect is consistent with the approximately $8-10$ times lower antiproton intensities compared to the proton intensities.

\section{Beam in the Tevatron}

It is known that beam can exist between beam bunches and in the beam abort gaps. Any DC beam is cleaned by 

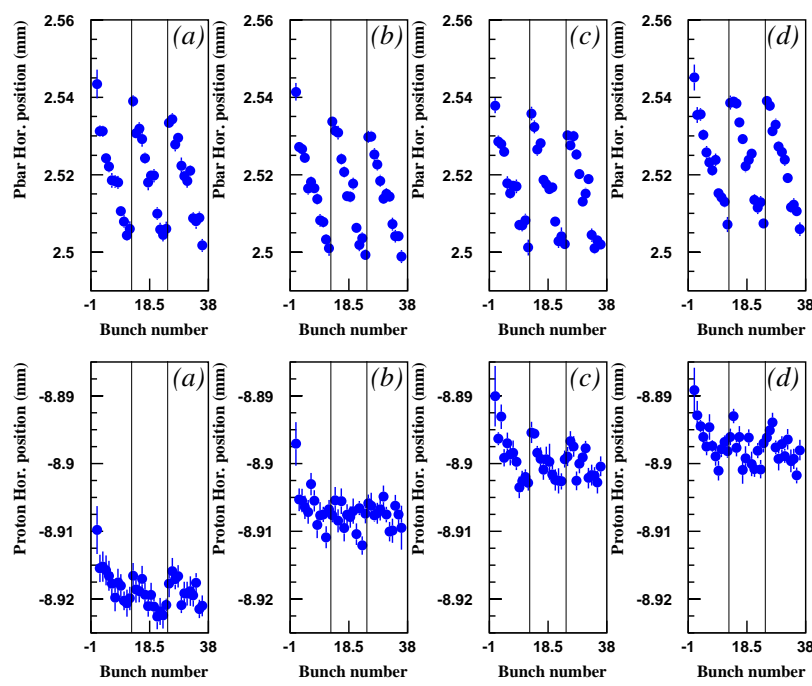

Figure 5: Horizontal positions of each of the 36 bunches for antiprotons (top) and protons (bottom), where (a, b, c, d) give the averaged positions for $0-20,20-40,40-60$ and 60 80 minutes after the start of collisions for a collider store.

the Tevatron Electron Lens (TEL) [9]. The Synclite system has been configured to allow automatic monitoring of any DC beam. A $670 \mathrm{~ns}$ gate width timed to look within a beam abort gap is used and the gain is increased by a factor of about 22 by running the MCP in the image intensifier at much higher voltage. Also 100 images are summed to improve $\mathrm{S} / \mathrm{N}$ since more noise appears when running at high MCP voltages. Typical projections of the background subtracted images are given in Figure 6.

With the TEL off, about $6 \times 10^{7}$ protons were observed within the $670 \mathrm{~ns}$ window. This is less than $\frac{1}{1000}$ th of the intensity of a single proton bunch. Assuming a uniform DC beam in the Tevatron, this translates to about $2 \times 10^{9}$ protons in total in the DC beam, or about $0.03 \%$ of the total number of protons in the 36 bunches. The transverse sigmas of the beam in the abort gap have large uncertainties due to poor $\mathrm{S} / \mathrm{N}$. The central location of the DC beam is found to be at the same vertical location as the normal bunches while the horizontal location is $1 \mathrm{~mm}$ closer to the Tevatron center. Work is being done to increase the gain and $\mathrm{S} / \mathrm{N}$.

\section{CONCLUSIONS}

Synclite provides a non-destructive method to measure the 2-dimensional transverse profiles of the high energy beam in the Tevatron. The system is still being calibrated but already interesting beam physics issues can be studied using this instrument.

\section{Acknowledgments}

We wish to thank S. Pordes and E. Lorman for their help and efforts on Synclite. We also want to thank P. Lebrun,

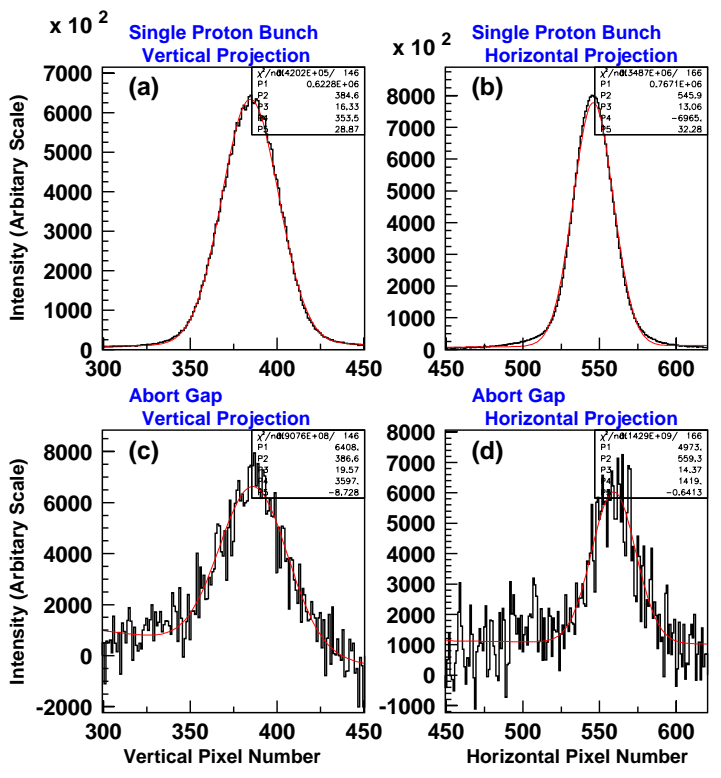

Figure 6: $(\mathrm{a}, \mathrm{b})$ Vertical and horizontal projections of the background subtracted image for a single $1.8 \times 10^{11}$ proton bunch at normal MCP high voltage; (c,d) Vertical and horizontal projections of the background subtracted image for a 670 ns time interval in the beam abort gap. The projections are fit with a Gaussian signal and a linear background function.

S. Panacek, T. Bolshakov, K. Genser, A. Slaughter, A. Jansson, V. Shiltsev and the whole SDA team for providing help with the data analysis and easy access to the data.

\section{REFERENCES}

[1] R. Coisson, "Angular-spectral distribution and polarization of synchrotron radiation from a 'short' magnet", Phys. Rev. A 20, 1979, pp.524-528.

[2] A. A. Hahn and P. Hurh, "Results from a Prototype Beam Monitor in the Tevatron Using Synchrotron Light", in 1992 IEEE Particle Accelerator Conference, 1991, pp.1177-1179.

[3] A. A. Hahn and P. Hurh, "Results from an Imaging Beam Monitor in the Tevatron Using Synchrotron Light", in 15th Int. Conf. on High Energy Accelerators, Ed. by J. Rossbach, Hamburg, Germany July 20-24 1992, pp.248-253, Int. J. Mod. Phys. A (Proc. Suppl.) 2A, 1993.

[4] A. Slaughter et al., "Store Data Analysis for Run II in the Fermilab Accelerator Complex", TPPB071, these proceedings.

[5] A. Jansson et al., "Collimator Scans to Measure Tevatron Emittance", Tevatron beam study report, Jan. 21, 2003, Beams-docdb-393.

[6] W. Blokland et al., "A new Flying Wire System for the Tevatron”, PAC‘97.

[7] A. Slaughter et al., "Tevatron Run II Luminosity, Emittance and Collision Point Size", TPPB070, these proceedings.

[8] http://home.fnal.gov/ cheung/synclite/ 
[9] X. Zhang et al., "The Special Applications of Tevatron Electron Lens in Collider Operation", TPPB076, these proceedings. 